\title{
Diffusion Measure Changes of Substantia Nigra Subregions and the Ventral Tegmental Area in Newly Diagnosed Parkinson's Disease
}

\author{
Jae-Hyuk Shim and Hyeon-Man Baek* \\ Department of Health Sciences and Technology, GAIHST, Gachon University, Incheon 21999, Korea
}

\begin{abstract}
Historically, studies have extensively examined the basal ganglia in Parkinson's disease for specific characteristics that can be observed with medical imaging. One particular methodology used for detecting changes that occur in Parkinson's disease brains is diffusion tensor imaging, which yields diffusion indices such as fractional anisotropy and radial diffusivity that have been shown to correlate with axonal damage. In this study, we compare the diffusion measures of basal ganglia structures (with substantia nigra divided into subregions, pars compacta, and pars reticula), as well as the diffusion measures of the diffusion tracts that pass through each pair of basal ganglia structures to see if significant differences in diffusion measures can be observed in structures or tracts in newly diagnosed Parkinson's disease patients. Additionally, we include the ventral tegmental area, a structure connected to various basal ganglia structures affected by dopaminergic neuronal loss and have historically shown significant alterations in Parkinson's disease, in our analysis. We found significant fractional anisotropy differences in the putamen, and in the diffusion tracts that pass through pairs of both substantia nigra subregions, subthalamic nucleus, parabrachial pigmental nucleus, ventral tegmental area. Additionally, we found significant radial diffusivity differences in diffusion tracts that pass through the parabrachial nucleus, putamen, both substantia nigra subregions, and globus pallidus externa. We were able to find significant diffusion measure differences in structures and diffusion tracts, potentially due to compensatory mechanisms in response to dopaminergic neuronal loss that occurs in newly diagnosed Parkinson's disease patients.
\end{abstract}

Key words: Parkinson’s disease, Basal ganglia, MRI, Diffusion tractography

\section{INTRODUCTION}

Basal ganglia structures such as the subthalamic nucleus (STN) and substantia nigra (SN) have been crucial targets for studying Parkinson's disease (PD). It has been well established that the death of dopaminergic neurons commonly occurs in the substantia nigra of clinical PD patients [1], which in turn contributes to the dysfunction of downstream nigrostriatal pathways (putamen, GPe, STN, GPi/SNr, thalamus, motor cortex) related to movement dis-

\footnotetext{
Submitted July 26, 2021, Revised September 13,2021 Accepted September 22, 2021

${ }^{*}$ To whom correspondence should be addressed. TEL: 82-32-899-6678, FAX: 82-32-899-6677 e-mail:hmbaek98@gachon.ac.kr
}

orders [2]. However, most reviews reported axonal degeneration starting from $50 \%$ of SN dopamine neurons to $70 \%$ before motor signs appear, making it vital to detect neuronal loss in the $\mathrm{SN}$ before PD diagnosis. Additionally, there are various diseases with similar motor symptoms that are found in PD such as multiple system atrophy and tremor, making it desirable to distinguish PD from other motor diseases by observing biomarkers visible in $\mathrm{SN}$ $[3,4]$. Examining how $\mathrm{SN}$ biomarkers change under treatments such as L-DOPA and deep brain stimulation can help explain the results of experiments regarding the effectiveness of treatments or conflicting results that arise due to side effects of treatments $[5,6]$.

Diffusion tensor imaging (DTI) has been used as a common MRI sequence to measure diffusion indices such as fractional anisotropy (FA), axial diffusivity (AD), radial diffusivity (RD), and mean diffusivity (MD), which describe random Brownian motion
Copyright ( Experimental Neurobiology 2021. www.enjournal.org
This is an Open Access article distributed under the terms of the Creative Commons Attribution Non-Commercial License (http://creativecommons.org/licenses/by-nc/4.0) which permits unrestricted non-commercial use, distribution, and reproduction in any medium, provided the original work is properly cited. 
of water molecules. Various studies have shown correlations between significantly low levels of diffusion measures and alterations in white matter microstructural integrity. Increases in RD have been shown to indicate fibers with significant demyelination while healthy, myelinated fibers were shown to have high FA and low RD $[7,8]$. Additionally, studies showed that decreased AD can indicate axonal injury, and increased MD can describe microstructural damages that occur in necrosis $[9,10]$. As such, many studies have compared diffusion measures between PD patients and healthy controls (HC) to observe whether the death of dopaminergic neurons in $\mathrm{SN}$ can be traced to significant changes in diffusion measures $[11,12]$.

While the focus of diffusion weighted results are generally on white matter tract integrity, diffusion indices have also been used to indicate significant changes in deep gray matter structures in various neurodegenerative disorders. As breakdown of microstructures in grey matter structures occur, certain diffusion indices such as MD can indicate increase in net diffusion due to fewer obstacles impeding diffusion, as opposed to diffusion in a particular direction [13]. Meta-analyses and review articles of PD DTI studies showed that studies were able to find significant changes in diffusion measures of various grey matter structures, particularly in the putamen and the thalamus. While many studies of DTI measures in PD SN, where dopaminergic neuronal loss occurs, were able to find significant differences in DTI measures of PD and HC SN, there were also conflicting results that suggest the use of DTI measures as PD biomarkers may be inconsistent [14].

In order to clarify whether DTI measures can be used for identifying abnormalities of the SN normally found in PD, various studies have attempted to replicate significant differences of DTI measures between PD and HC SN [15]. However, inconsistent results between several studies shifted the scope of finding potential significant DTI differences between SN down to SN subregions particularly in the pars compacta $\mathrm{SN}$ subregion $(\mathrm{SNc})$ where the loss of dopaminergic neurons occurs $[15,16]$. Most studies showed conflicting results regardless of the comparison between SN subregions, but such issues may have been attributed to inconsistencies in delineating SN into SNc and $\mathrm{SNr}$ due to their small size and lack of defining borders [11].

In this study, we compare the fractional anisotropy (FA), quantitative anisotropy (QA), mean diffusivity (MD), axial diffusivity (AD), radial diffusivity (RD), of regions defined by the CIT168 atlas where it is either rich in dopaminergic neurons or is the target of dopaminergic innervation between PD and HC. We delineate structures from the CIT168 atlas such as putamen, caudate, nucleus accumbens (NA), globus pallidus externa (GPe), globus pallidus interna $(\mathrm{GPi})$, substantia nigra pars compacta $(\mathrm{SNc})$, substantia nigra pars reticula (SNr), and subthalamic nucleus (STN) [17]. Additionally, as part of the CIT168 atlas, we delineate parabrachial pigmented nucleus (PBP) and ventral tegmental area (VTA), two structures part of the mesocorticolimbic pathway responsible for reward and motivation. While not directly affected by dopaminergic neuronal loss in the SNc, VTA, and PBP are inspected for significant differences in diffusion measures due to the associated neuronal and morphological changes that can occur in PD VTA and PBP. In addition, we generate diffusion tractography between each segmented structure for comparing DTI measures of white matter tracts that pass through each structure. Assessing diffusion measures of tracts representing pathways affected by PD may show more conclusive evidence of disrupted dopaminergic connectivity.

\section{MATERIALS AND METHODS}

\section{Subjects}

$65 \mathrm{HC}$ and $65 \mathrm{PD}$ subjects used in the preparation of this article were recruited from the Parkinson's Progression Markers Initiative (PPMI) database (www.ppmi-info.org/data) [18]. For up-todate information on the study, visit www.ppmi-info.org. Each PD subject was assessed for PD using Part III of Movement Disorder Society-sponsored revision of the Unified Parkinson's Disease Rating Scale (MDS-UPDRS III), observation of dopaminergic neurons through dopamine transporter SPECT scans, and displaying motor symptoms such as resting tremor or bradykinesia. Assessment of PD was done before any subjects were administered PD medication that can interfere with PD symptom testing. All subjects involved in this research were tested negative for neurological disorders apart from PD. Group demographics and clinical characteristics are shown in Table 1. All subjects provided written informed consent to share each subject's unidentified clinical data with investigators.

\section{MRI data acquisition}

MRI data of HC and PD subjects used in this study was obtained from the PPMI database (www.ppmi-info.org/data). Diffusion MRI images were acquired using standard protocols on 3T scanners at approximately 11 blinded PPMI imaging sites. T1-weighted images were acquired using 3D T1-weighted MPRAGE sequence (echo time $(\mathrm{TE})=90 \mathrm{~ms}$, repetition time $(\mathrm{TR})=2,300 \mathrm{~ms}, 1 \mathrm{~mm}^{3}$ resolution) and DTI images were acquired using $2 \mathrm{D}$ single-shot echo-planar DTI sequence (TE $=88 \mathrm{~ms}, \mathrm{TR}=900 \mathrm{~ms}, 2 \mathrm{~mm}^{3}$ resolution, 72 slices, flip angle $=90^{\circ}, 64$ gradient directions, $b$-value $=1,000$ $\mathrm{s} / \mathrm{mm}^{2}$ ). More details regarding MRI sequence information can be found in the PPMI MRI technical operations manual (https:// 
Table 1. Group characteristics of PD and HC

\begin{tabular}{lccc}
\multicolumn{1}{c}{ Group } & HC $(\mathbf{n}=\mathbf{6 5})$ & PD (n=65) & p-value \\
\hline Age (mean \pm SD) & $58.7 \pm 11.1$ & $60.5 \pm 9.6$ & 0.316 \\
Sex (male/female) & $44 / 21$ & $43 / 22$ & $27 / 38$ \\
Dominant side (left/right) & & $15.2 \pm 3.1$ & 0.186 \\
Education years (mean \pm SD) & $15.5 \pm 3.0$ & $58.4 \pm 10.2$ & $6.4 \pm 6.9$ \\
Age onset (years) & & $547.0 \pm 264.0$ & $27.7 \pm 1.8$ \\
Duration of disease (months) & & $19.5 \pm 8.9$ & 0.509 \\
Total levodopa equivalent daily dose & $28.3 \pm 1.2$ & $<0.001$ \\
MoCA (mean \pm SD) & $0.7 \pm 1.7$ & & \\
MDS-UPDRS III Score (mean \pm SD) & & & \\
\hline
\end{tabular}

HC, healthy controls; PD, parkinson's disease; SD, standard deviation; MoCA, Montreal Cognitive Assessment Test Scoring; MDS-UPDRS III, Movement Disorder Society-sponsored revision of the Unified Parkinson's Disease Rating Scale.

www.ppmi-info.org/wp-content/uploads/2017/06/PPMI-MRIOperations-Manual-V7.pdf).

\section{Image processing}

Prior to segmentation and diffusion fiber reconstruction, a series of preprocessing steps were performed on each subject's DTI image to correct for errors such as eddy currents and head motion. Through various programs provided by MRtrix3, DTI images went through denoising (dwidenoise), Gibbs ringing removal (dwidenoise), motion and distortion correction (dwifslreproc), bias field correction (dwibiascorrect), and resampled to $1 \mathrm{~mm}^{3}$ isotropic resolution (mrgrid) [19,20].

Atlas segmentation of CIT168 structures, putamen, caudate, nucleus accumbens (NA), globus pallidus external (GPe), globus pallidus internal (GPi), substantia nigra pars compacta $(\mathrm{SNc})$, substantia nigra pars reticula $(\mathrm{SNr})$, subthalamic nucleus (STN), parabrachial pigmented nucleus (PBP), ventral tegmental area (VTA), was done through the default Lead-DBS Lead Connectome pathway [21]. First, each subject's b0 image extracted from DTI images is co-registered to the T1w image using SPM12 (https:// www.fil.ion.ucl.ac.uk/spm/software/spm 12). Following the coregistration, the co-registered $\mathrm{T} 1 \mathrm{w}$ images are normalized using a modified version of advanced normalization tools (ANTs) to the MNI ICBM 2009b template space in which the CIT168 atlas is preregistered to. Inverse warps generated from normalization and co-registration are applied on the CIT168 atlas to warp the atlas to the subject's diffusion space. The quality of each co-registration and normalization were visually inspected using edge detectionbased wireframes and atlas segmentations overlaid on top of coregistered images generated by Lead-DBS.

\section{Diffusion processing}

Lead Connectome, a MATLAB-based structural connectomic analysis pipeline that utilizes DSI studio for generalized Q-ball imaging (GQI) for reconstruction and deterministic fiber tracking, was used to generate a structural connectome for each subject [22]. 400,000 tracts were generated using a step size of $0.5 \mathrm{~mm}$, an angular threshold of $75^{\circ}$, minimum length of $10 \mathrm{~mm}$, and maximum length of $300 \mathrm{~mm}$. DSI studios statistics tool was used to obtain values of diffusion network measures (FA, QA, MD, AD, RD) in segmented CIT168 structures, and the DSI studio's connectivity matrix tool was used to obtain diffusion network measures of fiber tracts that connect each pair of segmented structures.

\section{Statistical analysis}

Group characteristics, age, education years, and MoCA test scores were tested for significant differences using student's t-test to ensure that group differences of diffusion measures were not influenced by differences of group characteristics. Volumes and diffusion measures of segmented CIT168 structures were compared between all HC and PD using student's t-test. The BenjaminiHochberg false discovery rate (FDR) procedure was done on volumetric and diffusion measure comparisons with significance set to $\mathrm{p}<0.05$ to correct for false positives when conducting multiple comparisons. The same methodology using student's t-test and FDR was done to find significant differences (significance at $\mathrm{p}<0.05)$ in diffusion measures of PD and $\mathrm{HC}$ diffusion fiber tracts between each segmentation pair.

\section{RESULTS}

CIT168 structures that were segmented using PPMI HC and PD diffusion images with Lead-DBS are shown in Fig. 1. Demographics and clinical characteristics of $\mathrm{HC}$ and PD subjects were compared for significant differences using student's t-test. MDSUPDRS III scores showed significant differences $(\mathrm{p}<0.001)$ between HC and PD, as shown in Table 1.

The student's t-test was used to compare each volume and dif- 


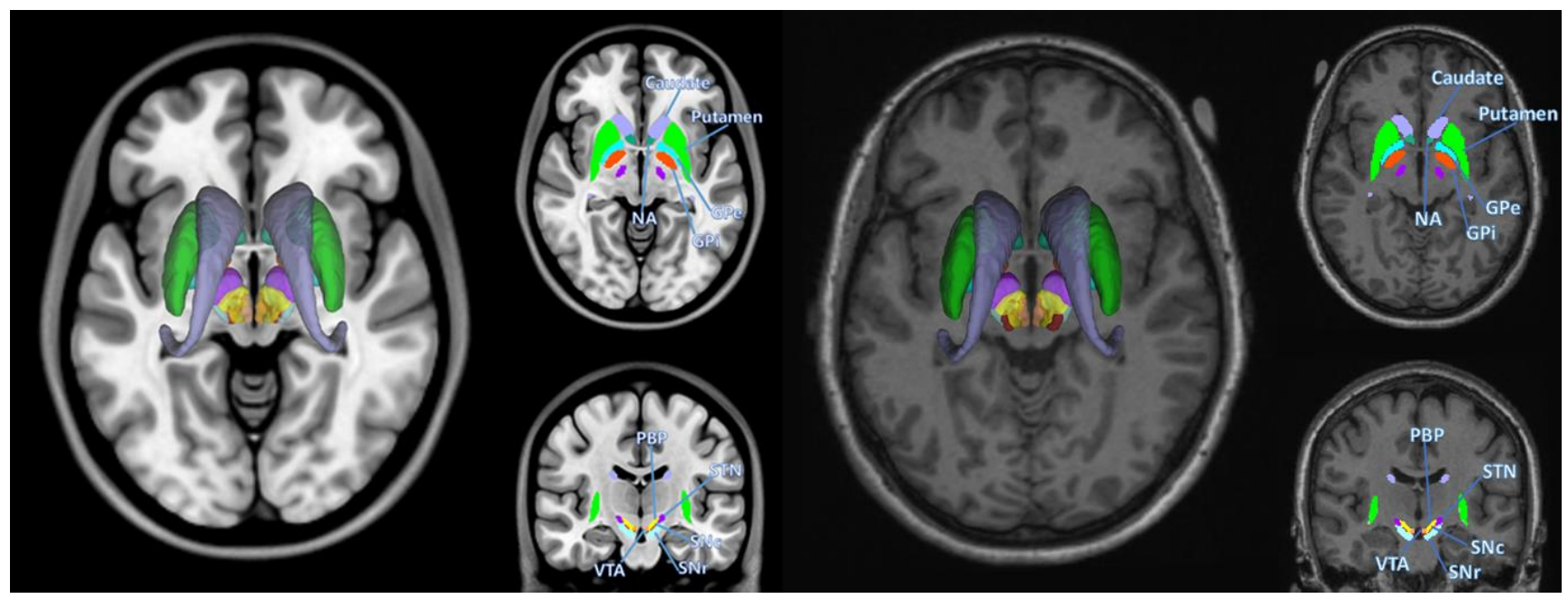

Fig. 1. Basal ganglia segmentation involved in Parkinson's disease. Left represents segmentations overlaid on top of MNI template, right represents an example segmentation of a PD subject used in this study (Patient 3127), overlaid on the same subject's T1w image. Structures segmented are left and right putamen, caudate, nucleus accumbens (NA), globus pallidus externus (GPe), globus pallidus internus (GPi), substantia nigra pars compacta (SNc), substantia nigra pars reticula (SNr), subthalamic nucleus (STN), parabrachial pigmented nucleus (PBP), ventral tegmental area (VTA).

Table 2. Volumetric differences of HC and PD basal ganglia, PBP,VTA segmentations

\begin{tabular}{lrrrrrrrrrrr}
\hline & & Putamen & Caudate & NA & GPe & GPi & SNr & SNc & STN & PBP & VTA \\
\hline \multirow{2}{*}{ HC volume } & L & $4,697.14$ & $4,475.98$ & 412.98 & 781.88 & 436.63 & 48.28 & 475.05 & 265.08 & 221.18 & 122.38 \\
& R & $4,957.72$ & $4,730.66$ & 492.94 & 856.49 & 482.18 & 38.52 & 529.46 & 265.75 & 232.14 & 116.78 \\
PD volume & L & $4,857.18$ & $4,689.72$ & 429.86 & 801.26 & 452.18 & 49.22 & 497.63 & 274.35 & 233.11 & 127.18 \\
& R & $5,121.98$ & $4,950.31$ & 513.48 & 882.75 & 500.05 & 40.83 & 550.89 & 278.95 & 245.28 & 121.12 \\
p & L & 0.32 & 0.32 & 0.32 & 0.44 & 0.32 & 0.73 & 0.31 & 0.32 & 0.27 & 0.36 \\
& R & 0.33 & 0.32 & 0.32 & 0.36 & 0.32 & 0.32 & 0.32 & 0.31 & 0.27 & 0.36 \\
\hline
\end{tabular}

Comparison of average volume (in $\mathrm{mm}^{3}$ ) between ROIs segmented using HC (healthy control) images and PD (Parkinson's disease) images. Each comparison was done through a t-test, and corrected for false positives through FDR correction. p-values shown in this table are p-values adjusted from FDR correction. No significant differences $(\mathrm{p}<0.05)$ were found between control volumes and patient volumes.

fusion measure (FA, QA, MD, AD, RD) of segmented CIT168 structures between $\mathrm{HC}$ and PD as shown in Table 2 and Table 3 . The Benjamini-Hochberg procedure was used to control for false discovery rate (FDR) with significance set to $\mathrm{p}<0.05$. Structures showed no significant group differences in volume of $\mathrm{HC}$ and $\mathrm{PD}$ segmentations as shown in Table 2. Structures with significant diffusion measure differences were putamen (FA, MD, AD, RD) and GPi (MD, RD) but post FDR correction showed significant differences were limited to MD and RD of the putamen.

The student's t-test was also used to compare the diffusion measures (FA, QA, MD, AD, RD) and fiber tract counts of connectivity between each CIT168 structure pair segmented with Lead-DBS. The Benjamini-Hochberg procedure was used to control for false discovery rate (FDR) with significance set to $\mathrm{p}<0.05$. No connectivity pair showed significant differences in fiber tract counts, MD, $\mathrm{AD}$, and QA post FDR correction. Only right hemisphere connectivity pairs showed significant difference between $\mathrm{HC}$ and PD diffusion measures. 8 connectivity pairs showed significant differences in FA and 4 connectivity pairs showed significant differences in RD as shown in Table 4.

\section{DISCUSSION}

For our study, we utilized diffusion MRI images to compare diffusion measures of CIT168 structures influenced by the death of dopaminergic neurons. We mainly focused on the significant differences of diffusion measures in $\mathrm{SN}$ substructures, $\mathrm{SNc}$ and $\mathrm{SNr}$, based on reports of DTI alterations being localized to SNc where the loss of dopaminergic neurons occurs. Additionally, we investigated the diffusion measures of diffusion fiber tracts that passed through pairs of CIT168 structures to observe how diffusion fibers change under PD. Our results revealed that after correcting for false discovery rate, the only structure that showed significant differences in diffusion measures was Putamen. SN substructures 
Table 3. Diffusion measure differences of the PD and HC basal ganglia, VTA, PBP

\begin{tabular}{|c|c|c|c|c|c|c|c|c|c|c|c|}
\hline \multirow{2}{*}{ Region } & & \multicolumn{2}{|c|}{ QA } & \multicolumn{2}{|c|}{ FA } & \multicolumn{2}{|c|}{ MD } & \multicolumn{2}{|c|}{$\mathrm{AD}$} & \multicolumn{2}{|c|}{$\mathbf{R D}$} \\
\hline & & HC & PD & HC & PD & HC & PD & HC & PD & HC & PD \\
\hline \multirow[t]{2}{*}{ Putamen } & $\mathrm{L}$ & 0.039 & 0.040 & 0.275 & 0.285 & 0.723 & 0.726 & 0.933 & 0.946 & 0.618 & 0.617 \\
\hline & $\mathrm{R}$ & 0.040 & 0.042 & $0.260^{\star}$ & $0.280^{*}$ & 0.780 & 0.746 & 0.997 & 0.974 & 0.672 & 0.632 \\
\hline \multirow[t]{2}{*}{ Caudate } & $\mathrm{L}$ & 0.040 & 0.038 & 0.204 & 0.194 & 1.213 & 1.363 & 1.418 & 1.565 & 1.110 & 1.262 \\
\hline & $\mathrm{R}$ & 0.038 & 0.036 & 0.182 & 0.176 & 1.376 & 1.492 & 1.580 & 1.692 & 1.274 & 1.392 \\
\hline \multirow[t]{2}{*}{ Nucleus accumbens } & $\mathrm{L}$ & 0.053 & 0.059 & 0.207 & 0.218 & 0.798 & 0.808 & 0.956 & 0.980 & 0.718 & 0.722 \\
\hline & $\mathrm{R}$ & 0.054 & 0.054 & 0.206 & 0.206 & 0.803 & 0.834 & 0.960 & 0.997 & 0.724 & 0.752 \\
\hline \multirow[t]{2}{*}{ Globus pallidus external } & $\mathrm{L}$ & 0.032 & 0.035 & 0.333 & 0.351 & 0.713 & 0.727 & 0.959 & 0.992 & 0.590 & 0.594 \\
\hline & $\mathrm{R}$ & 0.038 & 0.042 & 0.338 & 0.355 & 0.777 & 0.756 & 1.056 & 1.047 & 0.638 & 0.611 \\
\hline \multirow[t]{2}{*}{ Globus pallidus internal } & $\mathrm{L}$ & 0.048 & 0.048 & 0.425 & 0.429 & 0.687 & 0.699 & 1.006 & 1.029 & 0.527 & 0.534 \\
\hline & $\mathrm{R}$ & 0.051 & 0.053 & 0.426 & 0.435 & 0.738 & 0.720 & 1.085 & 1.074 & 0.565 & 0.543 \\
\hline \multirow[t]{2}{*}{ Substantia Nigra pars compacta } & $\mathrm{L}$ & 0.061 & 0.060 & 0.435 & 0.450 & 0.778 & 0.778 & 1.150 & 1.163 & 0.592 & 0.586 \\
\hline & $\mathrm{R}$ & 0.063 & 0.061 & 0.430 & 0.452 & 0.769 & 0.764 & 1.132 & 1.143 & 0.587 & 0.574 \\
\hline \multirow[t]{2}{*}{ Substantia Nigra pars reticular } & $\mathrm{L}$ & 0.067 & 0.066 & 0.556 & 0.568 & 0.756 & 0.756 & 1.241 & 1.257 & 0.515 & 0.506 \\
\hline & $\mathrm{R}$ & 0.062 & 0.062 & 0.513 & 0.540 & 0.812 & 0.790 & 1.277 & 1.277 & 0.580 & 0.548 \\
\hline \multirow{2}{*}{ Subthalamic nucleus } & $\mathrm{L}$ & 0.056 & 0.055 & 0.520 & 0.521 & 0.706 & 0.688 & 1.105 & 1.083 & 0.507 & 0.491 \\
\hline & $\mathrm{R}$ & 0.057 & 0.055 & 0.491 & 0.501 & 0.741 & 0.704 & 1.135 & 1.090 & 0.544 & 0.511 \\
\hline \multirow[t]{2}{*}{ Parabrachial pigmented nucleus } & $\mathrm{L}$ & 0.059 & 0.055 & 0.445 & 0.441 & 0.712 & 0.712 & 1.048 & 1.039 & 0.544 & 0.549 \\
\hline & $\mathrm{R}$ & 0.058 & 0.054 & 0.436 & 0.444 & 0.727 & 0.709 & 1.062 & 1.035 & 0.559 & 0.546 \\
\hline \multirow[t]{2}{*}{ Ventral tegmental area } & $\mathrm{L}$ & 0.077 & 0.072 & 0.470 & 0.475 & 0.733 & 0.706 & 1.086 & 1.054 & 0.556 & 0.532 \\
\hline & $\mathrm{R}$ & 0.076 & 0.072 & 0.450 & 0.464 & 0.749 & 0.755 & 1.092 & 1.106 & 0.578 & 0.579 \\
\hline
\end{tabular}

Average diffusion measures of left and right basal ganglia structures. Significant differences and p-values were calculated using student t-tests, with pvalues adjusted for multiple corrections using FDR correction. Bolded values represent diffusion measures with significant difference ( $\mathrm{p}<0.05$ ), values with asterisk represent measures with significant difference after FDR correction. FA, Fractional Anisotropy; QA, Quantitative Anisotropy; MD, Mean Diffusivity; AD, Axial Diffusivity; RD, Radial Diffusivity.

Table 4. Diffusion measures of diffusion connectivity between segmented structures with significant differences

\begin{tabular}{|c|c|c|c|c|}
\hline \multicolumn{2}{|c|}{ Regions } & $\begin{array}{c}\mathrm{HC} \\
\text { averages }\end{array}$ & $\begin{array}{c}\text { PD } \\
\text { averages }\end{array}$ & p \\
\hline \multicolumn{5}{|c|}{ FA } \\
\hline \multirow[t]{4}{*}{$\mathrm{SNc}$} & $\mathrm{SNr}$ & 0.470 & 0.489 & 0.035 \\
\hline & STN & 0.470 & 0.490 & 0.021 \\
\hline & PBP & 0.466 & 0.486 & 0.021 \\
\hline & VTA & 0.457 & 0.478 & 0.035 \\
\hline \multirow[t]{2}{*}{$\mathrm{SNr}$} & PBP & 0.460 & 0.482 & 0.021 \\
\hline & VTA & 0.442 & 0.465 & 0.021 \\
\hline STN & PBP & 0.467 & 0.485 & 0.037 \\
\hline PBP & VTA & 0.461 & 0.482 & 0.037 \\
\hline \multicolumn{5}{|c|}{ RD } \\
\hline \multirow[t]{2}{*}{ Putamen } & $\mathrm{SNr}$ & 0.577 & 0.533 & 0.041 \\
\hline & PBP & 0.605 & 0.557 & 0.034 \\
\hline GPE & PBP & 0.604 & 0.557 & 0.040 \\
\hline SNc & PBP & 0.595 & 0.560 & 0.034 \\
\hline
\end{tabular}

All regions in this table are right hemisphere structures. HC and PD averages represent averages of diffusion measures FA and RD. p-values in this table are FDR corrected. with significant diffusion measure differences in diffusion tracts paired with PBP, GPe, GPi, VTA, and putamen.

A significant number of studies on group differences of brain structure diffusion measures have been done to observe DTI changes in PD $[14,15]$. Effects of dopamine loss due to death of dopaminergic neurons in SN causing imbalance of several other neurotransmitters in downstream motor systems and other structures have been illustrated through diffusion measure changes [23, 24]. A review paper that compiled multiple different non-hypothesis-driven, diffusion assessments of PD and controls showed multiple instances where studies found correlations between motor symptoms and diffusion measure changes [14]. While the majority of such studies found FA decreases and MD increases in regions that were analyzed, many studies showed that PD subjects had FA increases in the putamen, results which are in line with our significant FA increases in $\mathrm{PD}$ right putamen $(\mathrm{HC}=0.260, \mathrm{PD}=0.280$, $\mathrm{p}=0.020)[25,26]$. The putamen is a key structure in the motor system affected by Parkinson's, as it is directly connected to the SNc through dopaminergic neurons [27]. Additionally, as a result of dopaminergic neuronal loss, projections from the putamen to other basal ganglia structures such as GPe, STN, GPi, and SNr are abnormally altered, causing increased inhibition of downstream signals to structures such as centromedian (CM) and the motor 
cortex [27]. This in turn reduces the excitatory signals from the $\mathrm{CM}$ and the motor cortex to the putamen, which effectively creates a loop of increased motor cortex inhibition signals as putamen is less able to suppress inhibition signals to the motor cortex [28].

While there were significant differences in diffusion measures of the putamen, there were no significant differences in diffusion measures of both $\mathrm{SNc}$ and $\mathrm{SNr}$ after FDR correction. As the focal point of numerous PD studies, the SN has been a frequent region of interest for finding correlations between dopaminergic neuronal death and change in diffusion measures [14]. Various meta-analyses and reviews reported 2 studies with increased FA in SN, 11 with no significant FA changes, and 19 with significant FA decreases $[14,15]$. Therefore, conflicting diffusion measure results have led to further investigation towards the substructures of $\mathrm{SN}$, which consists of the posterior part of $\mathrm{SN}$ where dopaminergic neurons are located, referred to as $\mathrm{SNc}$, and the lateral part of SN referred to as $\mathrm{SNr}$ [29]. Due to dopaminergic neuronal death being localized to SNc and excitatory signals to $\mathrm{SNr}$ being less inhibited by loss of dopamine, it is possible that observing $\mathrm{SN}$ as a whole can lead to inconsistencies in significant diffusion measure differences [29]. Despite our results showing significant FA differences in both of the $\mathrm{SN}$ subregions, none of the results survived FDR correction. Our results were similar to other previous studies that reported no significant diffusion measure differences in either subregion of $\mathrm{SN}$, but multiple conflicting studies were also able to find significant FA differences in SN subregions [30, 31]. Inconsistencies between results of studies observing $\mathrm{SN}$ can be due to multiple factors regarding the type of PD subjects involved in studies and the consistencies between $\mathrm{SN}$ segmentations. PPMI subjects involved in the study consist of early diagnosed cases, averaging at 6.4 months of disease duration and consisting of mixed young-onset subjects (age $\leq 50)$ and standard onset subjects (age $>50$ ). A longitudinal study comparing FA of brain structures between types of PPMI subjects involving young onset, young controls, old onset, and old controls showed that young-onset subjects had significantly higher FA in multiple brain structures than young controls while older onset subjects had significantly lower FA in SN than old controls [14]. As such, the mixture of subject ages, duration of disease, and diagnosis could have influenced how significant the FA differences were in the subregions of $\mathrm{SN}$.

In our analysis of diffusion measures of basal ganglia diffusion tractography, we found several diffusion tracts that pass through pairs of basal ganglia structures with significant differences in FA and RD. Tractography that passed through pair combinations of basal ganglia structures, SNc, SNr, PBP, and VTA showed FA increases in PD subjects, and tractography that passed through $\mathrm{PBP}$ and either putamen, GPe, or $\mathrm{SNc}$ showed RD decreases in PD subjects ( $\mathrm{SNc}, \mathrm{SNr}, \mathrm{p}=0.035 ; \mathrm{SNc}$, STN, $\mathrm{p}=0.021 ; \mathrm{SNc}$, PBP, $\mathrm{p}=0.021$; $\mathrm{SNc}$, VTA, $\mathrm{p}=0.035$; $\mathrm{SNr}$, PBP, $\mathrm{p}=0.021$; $\mathrm{SNr}$, VTA, $\mathrm{p}=0.021$; STN, PBP, $p=0.037$; PBP, VTA, $p=0.037$ ). Diffusion tractography has been used prominently for detecting connectivity between regions of interest by simulating a rough estimate of white matter tracts through the diffusion of water [32]. As such, PD studies have utilized diffusion tractography to identify significant changes in tractography that might reflect the disruption of dopaminergic connectivity and its effects on the basal ganglia $[33,34]$. Reports of significant decreases in FA of diffusion tractography have been consistent throughout various studies, particularly in the nigrostriatal tract that connects the $\mathrm{SN}$ to the putamen via dopaminergic neurons $[14,33,34]$. However, our results showed no significant FA differences in tracts with any basal ganglia structure that pairs with the putamen, and the pairs of basal ganglia structures ( $\mathrm{SNc}$, $\mathrm{SNr}$, PBP, VTA) that did show significant FA differences were FA increases rather than the commonly reported FA decreases. There are several possible explanations for significant FA increases, including inflammation from dopaminergic neuronal loss and compensatory responses to structural connectivity changes [35]. VTA and PBP are part of the mesocorticolimbic reward and motivation system, which involves the A10 group of dopaminergic neurons situated laterally to the A9 group of dopaminergic neurons stemming from SNc [36]. Neurons passing through VTA have been shown to be connected to structures that are also connected to $\mathrm{SNc}$, and albeit at a slower rate, degenerate in PD similarly to SNc dopaminergic neurons [37]. Due to its close location from SNc, diffusion tracts that are shown to pass through VTA, PBP with SNc may show increased FA due to elevated levels of oxidative stress and neuroinflammation from dopamine degradation [3840]. Another potential explanation for the FA increase in VTA, PBP connectivity is enhanced activation of bidirectional neurons from the striatum to the VTA due to a disrupted regulatory system reliant on dopamine signaling [41].

$\mathrm{RD}$, which measures the diffusivity perpendicular to the tract, has been historically used for detecting demyelination and inflammation. An increase in RD has been correlated with demyelinated axons, as well as increased motor and memory dysfunctions $[42,43]$. However, our results using the PPMI dataset showed significantly decreased PD RD in tracts passing through the PBP, $\mathrm{SNr}, \mathrm{SNc}$, Putamen, and GPe (putamen, $\mathrm{SNr}, \mathrm{p}=0.041$; putamen, PBP, $\mathrm{p}=0.034$; GPe, PBP, $\mathrm{p}=0.040$; SNc, PBP, $\mathrm{p}=0.034$ ). Similar to our seemingly conflicting results regarding FA increases in $\mathrm{PD}$ diffusion tracts, the decrease in RD can be attributed to neural compensatory mechanisms observed in PD patients at the early stages of diagnosis. A study comparing diffusion measures of diffusion tracts in PD patients with early and progressive stages of 
PD showed that the lower RD observed in early PD patient white matter tracts became more comparable to that of $\mathrm{HC}$ white matter tracts at later stages of the disease [44]. The study suggests that the initial decreases of RD in PD were from compensations from dopaminergic deficiency which slowly phases out due to worsened symptoms and expended neural resources as the disease progresses, resulting in increased RD of white matter tracts [44].

There are various limitations of the methods used in the study that could have significantly affected the results. First, the PPMI data used in this study were acquired from 11 different sites, with their locations blinded for subject confidentiality, and potentially with different MRI vendors. While quality check from PPMI and preprocessing steps for diffusion images were done to correct for possible inhomogeneities that can arise due to different scanners, it is possible that our results were significantly affected regardless. Second, the normalization and coregistration methods used for atlas-based segmentations in the Lead-DBS pipeline while utilizes algorithms shown to have high dice-coefficient when segmenting the STN and GPi, may have minor inaccuracies in segmenting other regions of the basal ganglia. While all segmentations through this method were checked visually, it is possible that certain regions had minor inaccuracies, which could significantly affect DTI metrics due to the small size of the segmentations. Third, certain regions, such as VTA, PBP, and the inner boundaries of the two SN substructures, SNc and SNr do not express boundaries in T1w and diffusion weighted images. As such, visual inspection of such structures are limited to their general location given by the CIT168 atlas.

In this study, we were able to utilize the Lead-DBS segmentation pipeline and DSI studio diffusion tractography pipeline to compare diffusion measures of basal ganglia structures and the tracts that pass through the structures. We were able to find significant FA differences in the putamen, then find significant FA differences in tracts covering basal ganglia structures involved in PD, such as VTA, PBP, SNc, SNr, and STN. Our findings showed that similar to other studies on PD patients at earlier stages of the disease, the tracts passing through basal ganglia had significant diffusion measure differences that were contradictory to studies with PD at more advanced stages of the disease, likely due to compensatory mechanisms that slowly get weaker as the disease progresses. Future longitudinal studies on PD patients will be necessary to verify whether the pattern of diffusion measure changes in diffusion tracts as the disease progresses also occur in VTA and PBP diffusion tracts.

\section{ACKNOWLEDGEMENTS}

This research was supported by Brain Research Program through the National Research Foundation of Korea (NRF) funded by the Ministry of Science and ICT (NRF-2017M3C7A1044367).

\section{REFERENCES}

1. Surmeier DJ, Obeso JA, Halliday GM (2017) Selective neuronal vulnerability in Parkinson disease. Nat Rev Neurosci 18:101-113.

2. Poewe W, Seppi K, Tanner CM, Halliday GM, Brundin P, Volkmann J, Schrag AE, Lang AE (2017) Parkinson disease. Nat Rev Dis Primers 3:17013.

3. Wenning GK, Ben-Shlomo Y, Hughes A, Daniel SE, Lees A, Quinn NP (2000) What clinical features are most useful to distinguish definite multiple system atrophy from Parkinson's disease? J Neurol Neurosurg Psychiatry 68:434-440.

4. Schocke MF, Seppi K, Esterhammer R, Kremser C, Mair KJ, Czermak BV, Jaschke W, Poewe W, Wenning GK (2004) Trace of diffusion tensor differentiates the Parkinson variant of multiple system atrophy and Parkinson's disease. Neuroimage 21:1443-1451.

5. Vingerhoets FJ, Villemure JG, Temperli P, Pollo C, Pralong E, Ghika J (2002) Subthalamic DBS replaces levodopa in Parkinson's disease: two-year follow-up. Neurology 58:396-401.

6. Guttuso T Jr, Bergsland N, Hagemeier J, Lichter DG, Pasternak O, Zivadinov R (2018) Substantia nigra free water increases longitudinally in Parkinson disease. AJNR Am J Neuroradiol 39:479-484.

7. Salan T, Jacobs EL, Reddick WE (2017) A 3D model-based simulation of demyelination to understand its effects on diffusion tensor imaging. Annu Int Conf IEEE Eng Med Biol Soc 2017:3525-3528.

8. Chang EH, Argyelan M, Aggarwal M, Chandon TS, Karlsgodt KH, Mori S, Malhotra AK (2017) The role of myelination in measures of white matter integrity: combination of diffusion tensor imaging and two-photon microscopy of CLARITY intact brains. Neuroimage 147:253-261.

9. Gu L, Li J, Feng DF, Cheng ET, Li DC, Yang XQ, Wang BC (2013) Detection of white matter lesions in the acute stage of diffuse axonal injury predicts long-term cognitive impairments: a clinical diffusion tensor imaging study. J Trauma Acute Care Surg 74:242-247.

10. Winklewski PJ, Sabisz A, Naumczyk P, Jodzio K, Szurowska E, Szarmach A (2018) Understanding the physiopathology behind axial and radial diffusivity changes-what do we know? 
Front Neurol 9:92.

11. Schwarz ST, Abaei M, Gontu V, Morgan PS, Bajaj N, Auer DP (2013) Diffusion tensor imaging of nigral degeneration in Parkinson's disease: a region-of-interest and voxel-based study at $3 \mathrm{~T}$ and systematic review with meta-analysis. Neuroimage Clin 3:481-488.

12. Nagae LM, Honce JM, Tanabe J, Shelton E, Sillau SH, Berman BD (2016) Microstructural changes within the basal ganglia differ between Parkinson disease subtypes. Front Neuroanat 10:17.

13. Drake-Pérez M, Boto J, Fitsiori A, Lovblad K, Vargas MI (2018) Clinical applications of diffusion weighted imaging in neuroradiology. Insights Imaging 9: 535-547.

14. Zhang Y, Burock MA (2020) Diffusion tensor imaging in Parkinson's disease and Parkinsonian syndrome: a systematic review. Front Neurol 11:531993.

15. Deng XY, Wang L, Yang TT, Li R, Yu G (2018) A meta-analysis of diffusion tensor imaging of substantia nigra in patients with Parkinson's disease. Sci Rep 8:2941.

16. Hirata FCC, Sato JR, Vieira G, Lucato LT, Leite CC, Bor-SengShu E, Pastorello BF, Otaduy MCG, Chaim KT, Campanholo KR, Novaes NP, Melo LM, Gonçalves MR, do Nascimento FBP, Teixeira MJ, Barbosa ER, Amaro E Jr, Cardoso EF (2017) Substantia nigra fractional anisotropy is not a diagnostic biomarker of Parkinson's disease: a diagnostic performance study and meta-analysis. Eur Radiol 27:2640-2648.

17. Pauli WM, Nili AN, Tyszka JM (2018) A high-resolution probabilistic in vivo atlas of human subcortical brain nuclei. Sci Data 5:180063.

18. Parkinson Progression Marker Initiative (2011) The Parkinson progression marker initiative (PPMI). Prog Neurobiol 95:629-635.

19. Andersson JLR, Sotiropoulos SN (2016) An integrated approach to correction for off-resonance effects and subject movement in diffusion MR imaging. Neuroimage 125:10631078 .

20. Tournier JD, Smith R, Raffelt D, Tabbara R, Dhollander T, Pietsch M, Christiaens D, Jeurissen B, Yeh CH, Connelly A (2019) MRtrix3: a fast, flexible and open software framework for medical image processing and visualisation. Neuroimage 202:116137.

21. Horn A, Li N, Dembek TA, Kappel A, Boulay C, Ewert S, Tietze A, Husch A, Perera T, Neumann WJ, Reisert M, Si H, Oostenveld R, Rorden C, Yeh FC, Fang Q, Herrington TM, Vorwerk J, Kühn AA (2019) Lead-DBS v2: towards a comprehensive pipeline for deep brain stimulation imaging. Neuroimage 184:293-316.
22. Yeh FC, Wedeen VJ, Tseng WY (2010) Generalized q-sampling imaging. IEEE Trans Med Imaging 29:1626-1635.

23. Boonstra JT, Michielse S, Temel Y, Hoogland G, Jahanshahi A (2020) Neuroimaging detectable differences between Parkinson's disease motor subtypes: a systematic review. Mov Disord Clin Pract 8:175-192.

24. Pandya S, Zeighami Y, Freeze B, Dadar M, Collins DL, Dagher A, Raj A (2019) Predictive model of spread of Parkinson's pathology using network diffusion. Neuroimage 192:178-194.

25. Mole JP, Subramanian L, Bracht T, Morris H, Metzler-Baddeley C, Linden DE (2016) Increased fractional anisotropy in the motor tracts of Parkinson's disease suggests compensatory neuroplasticity or selective neurodegeneration. Eur Radiol 26:3327-3335.

26. Skidmore FM, Spetsieris PG, Anthony T, Cutter GR, von Deneen KM, Liu Y, White KD, Heilman KM, Myers J, Standaert DG, Lahti AC, Eidelberg D, Ulug AM (2015) A full-brain, bootstrapped analysis of diffusion tensor imaging robustly differentiates Parkinson disease from healthy controls. Neuroinformatics 13:7-18.

27. Galvan A, Devergnas A, Wichmann T (2015) Alterations in neuronal activity in basal ganglia-thalamocortical circuits in the Parkinsonian state. Front Neuroanat 9:5.

28. Hensel L, Hoffstaedter F, Caspers J, Michely J, Mathys C, Heller J, Eickhoff CR, Reetz K, Südmeyer M, Fink GR, Schnitzler A, Grefkes C, Eickhoff SB (2019) Functional connectivity changes of key regions for motor initiation in Parkinson's disease. Cereb Cortex 29:383-396.

29. Vaillancourt DE, Spraker MB, Prodoehl J, Abraham I, Corcos DM, Zhou XI, Comella CL, Little DM (2009) High-resolution diffusion tensor imaging in the substantia nigra of de novo Parkinson disease. Neurology 72:1378-1384.

30. Prakash BD, Sitoh YY, Tan LC, Au WL (2012) Asymmetrical diffusion tensor imaging indices of the rostral substantia nigra in Parkinson's disease. Parkinsonism Relat Disord 18:10291033.

31. Ofori E, Pasternak O, Planetta PJ, Burciu R, Snyder A, Febo M, Golde TE, Okun MS, Vaillancourt DE (2015) Increased free water in the substantia nigra of Parkinson's disease: a singlesite and multi-site study. Neurobiol Aging 36:1097-1104.

32. Lenglet C, Abosch A, Yacoub E, De Martino F, Sapiro G, Harel $\mathrm{N}$ (2012) Comprehensive in vivo mapping of the human basal ganglia and thalamic connectome in individuals using 7T MRI. PLoS One 7:e29153.

33. Zhang Y, Wu IW, Buckley S, Coffey CS, Foster E, Mendick S, Seibyl J, Schuff N (2015) Diffusion tensor imaging of the nigrostriatal fibers in Parkinson's disease. Mov Disord 30:1229- 
1236.

34. Cousineau M, Jodoin PM, Morency FC, Rozanski V, Grand'Maison M, Bedell BJ, Descoteaux M (2017) A testretest study on Parkinson's PPMI dataset yields statistically significant white matter fascicles. Neuroimage Clin 16:222233.

35. Faivre F, Sánchez-Catalán MJ, Dovero S, Bido S, Joshi A, Bezard E, Barrot M (2020) Ablation of the tail of the ventral tegmental area compensates symptoms in an experimental model of Parkinson's disease. Neurobiol Dis 139:104818.

36. Fu Y, Paxinos G, Watson C, Halliday GM (2016) The substantia nigra and ventral tegmental dopaminergic neurons from development to degeneration. J Chem Neuroanat 76(Pt B):98-107.

37. Caminiti SP, Presotto L, Baroncini D, Garibotto V, Moresco RM, Gianolli L, Volonté MA, Antonini A, Perani D (2017) Axonal damage and loss of connectivity in nigrostriatal and mesolimbic dopamine pathways in early Parkinson's disease. Neuroimage Clin 14:734-740

38. Kostuk EW, Cai J, Iacovitti L (2018) Regional microglia are transcriptionally distinct but similarly exacerbate neurodegeneration in a culture model of Parkinson's disease. J Neuro- inflammation 15:139.

39. Zhang J, Yang B, Sun H, Zhou Y, Liu M, Ding J, Fang F, Fan Y, Hu G (2016) Aquaporin-4 deficiency diminishes the differential degeneration of midbrain dopaminergic neurons in experimental Parkinson's disease. Neurosci Lett 614:7-15.

40. Pajares M, I Rojo A, Manda G, Boscá L, Cuadrado A (2020) Inflammation in Parkinson's disease: mechanisms and therapeutic implications. Cells 9:1687.

41. Haber SN (2014) The place of dopamine in the cortico-basal ganglia circuit. Neuroscience 282:248-257.

42. Song SK, Sun SW, Ramsbottom MJ, Chang C, Russell J, Cross AH (2002) Dysmyelination revealed through MRI as increased radial (but unchanged axial) diffusion of water. Neuroimage 17:1429-1436.

43. Theilmann RJ, Reed JD, Song DD, Huang MX, Lee RR, Litvan I, Harrington DL (2013) White-matter changes correlate with cognitive functioning in Parkinson's disease. Front Neurol $4: 37$.

44. Wen MC, Heng HS, Ng SY, Tan LC, Chan LL, Tan EK (2016) White matter microstructural characteristics in newly diagnosed Parkinson's disease: an unbiased whole-brain study. Sci Rep 6:35601. 